\title{
An unexpected and unusually complex Co-Mefenamate with nine octahedrally coordinated crystallographically independent cobalt atoms obtained at room temperature
}

\author{
G. Díaz de Delgado', M. Vivas-Arellano1, R.A. Toro², J.A. Henao², J.M. Delgado \\ ${ }^{1}$ Laboratorio de Cristalografia-LNDRX, Departamento de Química, Facultad de Ciencias, Universidad de Los Andes, Mérida 5101, \\ Venezuela, \\ ${ }^{2}$ Grupo de Investigación en Química Estructural (GIQUE), Escuela de Química, Facultad de Ciencias, Universidad Industrial de \\ Santander, Bucaramanga, Colombia.
}

gdiazdedelgado@gmail.com

Mefenamic acid (MefA) is one of the non-steroidal anti-inflammatory drugs (NSAID) commonly used in the treatment of mild to moderate pain. Some of its metal derivatives have shown greater pharmacological activity than mefenamic acid, in addition to fewer side effects of the acid in the digestive tract. With this in mind, it was considered of interest to prepare simple metal complexes of MefA. Reaction of $\mathrm{Co}\left(\mathrm{CH}_{3} \mathrm{COO}\right)_{2} \cdot 4 \mathrm{H}_{2} \mathrm{O}$ and sodium mefenamate (prepared from $\mathrm{NaOH}$ and MefA) in water at ambient conditions, produced a purple precipitate which was filtered and washed with $\mathrm{MeOH}$ :water. FT-IR indicated this was a Co-Mefenamic acid derivative. After a solubility study, the product was recrystallized from $N, N$-dimethylformamide (DMF) by slow evaporation at room temperature. Very small and thin pink needles were obtained after approximately 4 weeks. These crystals were characterized by ATR-IR spectroscopy and single crystal X-ray diffraction. This material crystallizes in a monoclinic $P 2_{1} / c$ (No. 14) unit cell with an unusually large volume: $a=15.9550(2) \AA, b=33.5553(11) \AA, c=31.6703(10) \AA, \beta=90.898(2)^{\circ}, V=16953.4(8) \AA^{3}, Z=4$.

Structure determination and refinement showed a complex structure based on a cluster of nine octahedrally coordinated crystallographically independent cobalt atoms, eight mefenamate ligands, six bridging hydroxyl groups, six DMF molecules, one $\mathrm{MeOH}$, three water molecules, and two carbonato moieties at the core of the cluster. $\mathrm{NaCO}_{3}$ was identified as an impurity in the $\mathrm{NaOH}$ used to prepare the Na-Mefenamate reagent. The mefenamate ligands coordinate in a bridging bidentate mode and exhibit intramolecular $\mathrm{N}-\mathrm{H} \cdots \mathrm{O}$ hydrogen bonds. Intermolecular $\mathrm{H}$-bonds occur between carboxylate oxygens, water, $\mathrm{DMF}, \mathrm{and} \mathrm{MeOH}$ molecules. Additional $\pi \cdots \pi$ and $\mathrm{C}-\mathrm{H} \cdots \pi$ interactions are important in stabilizing the structure. It is worth noting that only three

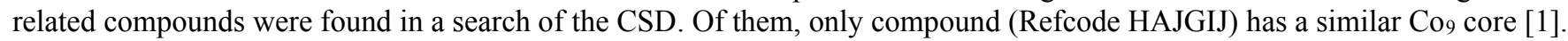

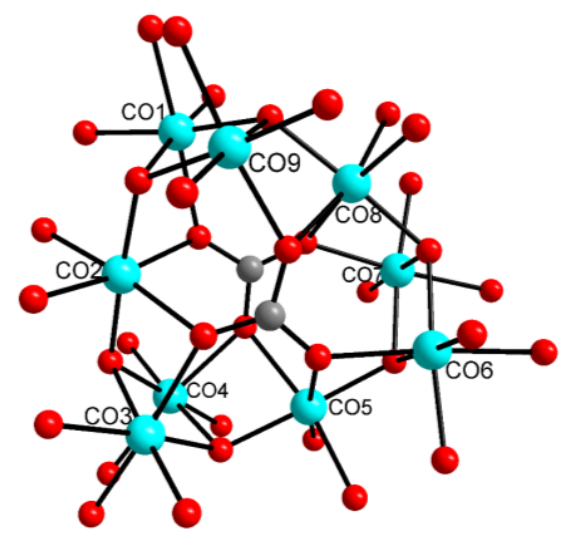

Figure 1. A view of the nonanuclear Co9O36 core in the Co-Mefenamate complex.

[1] Parsons, S., Brechin, E., Winpenny, R. \& Wood, P. A. (2004). HAJGIJ. CSD Communication.

Keywords: mefenamic acid; cobalt cluster; polynuclear complex; NSAIDs

The authors thank Vicerrectoría de Investigación y Extensión,Universidad Industrial de Santander (UIS) and Laboratorio de Rayos$X$-Parque Tecnológico Guatiguará, UIS, Colombia, for their support to the diffraction data collection facilities. Access to the CSD for ULA-Venezuela, is possible through the Frank H. Allen International Research \& Education Programme (FAIRE) from the CCDC. 